\title{
Determination of photocarrier density under continuous photoirradiation using spectroscopic techniques as applied to polymer: Fullerene blend films
}

Katsuichi Kanemoto, Hitomi Nakatani, Shinya Domoto

\begin{tabular}{|c|l|}
\hline Citation & Journal of Applied Physics, 116(16); 163103 \\
\hline Issue Date & $2014-10-27$ \\
\hline Type & Journal Article \\
\hline Textversion & publisher \\
\hline Right & $\begin{array}{l}\text { This article may be downloaded for personal use only. Any other use requires prior } \\
\text { permission of the author and AIP Publishing. The following article appeared in } \\
\text { Journal of Applied Physics and maybe found at https://doi.org/10.1063/1.4899609 }\end{array}$ \\
\hline URI & $\begin{array}{l}\text { http://dlisv03.media.osaka-cu.ac.jp/il/meta_pub/G0000438repository_10897550-11 } \\
6-16-163103\end{array}$ \\
\hline DOI & $10.1063 / 1.4899609$ \\
\hline
\end{tabular}

SURE: Osaka City University Repository

http://dlisv03.media.osaka-cu.ac.jp/il/meta_pub/G0000438repository

Katsuichi Kanemoto, Hitomi Nakatani, Shinya Domoto. (2014). Determination of photocarrier density under continuous photoirradiation using spectroscopic techniques as applied to polymer: Fullerene blend films. Journal of Applied Physics. 116, 163103. 


\section{Determination of photocarrier density under continuous photoirradiation using spectroscopic techniques as applied to polymer: Fullerene blend films}

Cite as: J. Appl. Phys. 116, 163103 (2014); https://doi.org/10.1063/1.4899609

Submitted: 30 April 2014 . Accepted: 14 October 2014 . Published Online: 27 October 2014

Katsuichi Kanemoto, Hitomi Nakatani, and Shinya Domoto
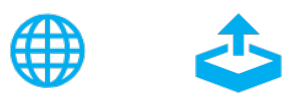

\section{ARTICLES YOU MAY BE INTERESTED IN}

Ultrafast carrier dynamics in thin-films of the topological insulator $\mathrm{Bi}_{2} \mathrm{Se}_{3}$

Applied Physics Letters 103, 151903 (2013); https://doi.org/10.1063/1.4824821

Sensitive monitoring of photocarrier densities in the active layer of a photovoltaic device with time-resolved terahertz reflection spectroscopy

Applied Physics Letters 110, 071108 (2017); https://doi.org/10.1063/1.4975631

Detection of current induced spin polarization in epitaxial $\mathrm{Bi}_{2} \mathrm{Te}_{3}$ thin film

Applied Physics Letters 110, 122403 (2017); https://doi.org/10.1063/1.4978691

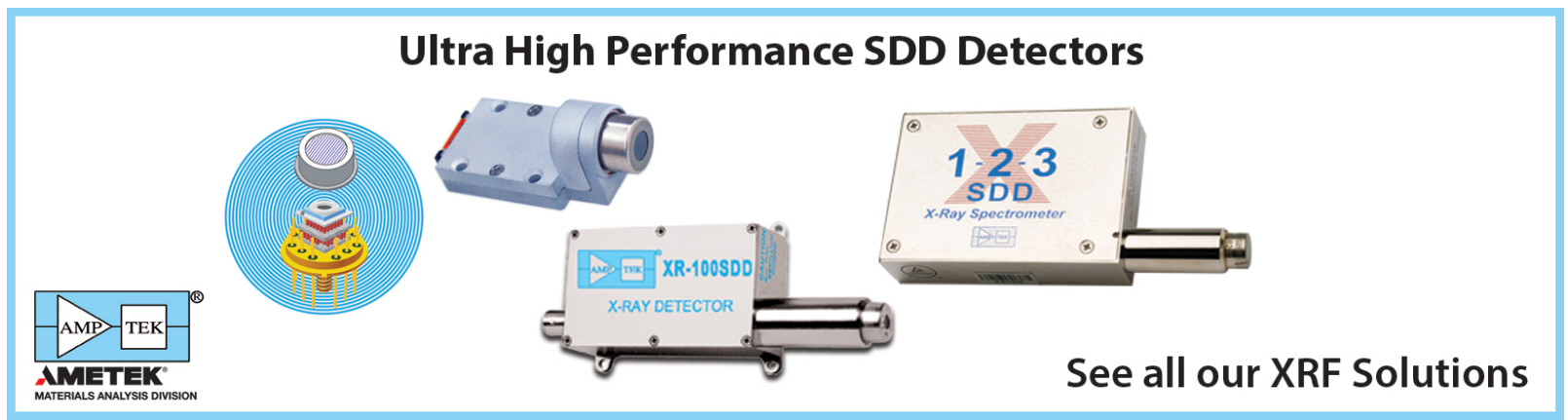




\title{
Determination of photocarrier density under continuous photoirradiation using spectroscopic techniques as applied to polymer: Fullerene blend films
}

\author{
Katsuichi Kanemoto, ${ }^{\text {a) }}$ Hitomi Nakatani, and Shinya Domoto \\ Department of Physics, Osaka City University, 3-3-138 Sugimoto, Sumiyoshi-ku, Osaka 558-8585, Japan
}

(Received 30 April 2014; accepted 14 October 2014; published online 27 October 2014)

\begin{abstract}
We propose a method to determine the density of photocarrier under continuous photoirradiation in conjugated polymers using spectroscopic signals obtained by photoinduced absorption (PIA) measurements. The bleaching signals in the PIA measurements of polymer films and the steadystate absorption signals of oxidized polymer solution are employed to determine the photocarrier density. The method is applied to photocarriers of poly (3-hexylthiophene) (P3HT) in a blended film consisting of P3HT and [6,6]-phenyl C61 butyric acid methyl ester (PCBM). The photocarrier density under continuous photoirradiation of $580 \mathrm{~mW} / \mathrm{cm}^{2}$ is determined to be $3.5 \times 10^{16} \mathrm{~cm}^{-3}$. Using a trend of the carrier density increasing in proportion to the square root of photo-excitation intensity, we provide a general formula to estimate the photocarrier density under simulated 1 sun solar irradiation for the P3HT: PCBM film of an arbitrary thickness. We emphasize that the method proposed in this study enables an estimate of carrier density without measuring a current and can be applied to films with no electrodes as well as to devices. (C) 2014 AIP Publishing LLC.

[http://dx.doi.org/10.1063/1.4899609]
\end{abstract}

\section{INTRODUCTION}

The photophysics of conjugated polymers has been intensely studied for the past three decades to understand their intriguing photophysical properties such as photoluminescence (PL) and photoconductivity. ${ }^{1-3}$ The excitons and photocarriers generated by photoexcitation are responsible for the photophysical properties and their photophysics have remained important subjects for study. The photophysics of photocarriers has particularly been intently studied because bulk-hetero junction (BHJ) organic solar cells based on polymer:fullerene films have attracted much attention as promising low cost solar cells. For enhancing the performance of BHJ solar cells towards practical applications, a deep understanding of the nature and dynamics of the photocarriers is required. ${ }^{4,5}$ Particularly, although theoretical approaches to explain the photophysical properties of the BHJ system have recently been developing, ${ }^{6-10}$ providing reliable experimental data to characterize the photocarriers in the BHJ structure are still key elements toward understanding the nature of the photocarriers.

Spectroscopic techniques are straightforward for characterizing the photophysical properties of photocarriers. They are particularly effective to examine the microscopic nature of photocarriers. ${ }^{11}$ The primary information provided by spectroscopic measurements is a spectrum and a lifetime of photoexcited states, and such data are used to analyze the electronic structure and dynamics of photocarriers. However, to our knowledge, a general method to estimate the density of photocarriers directly from spectroscopic measurements has not been proposed for solid conjugated polymers, even though this quantity is a very important parameter when discussing

\footnotetext{
${ }^{\text {a) }}$ Author to whom correspondence should be addressed. Electronic mail: kkane@sci.osaka-cu.ac.jp
}

the photophysics. For polymers in solution, the number of carriers (cations) generated by oxidation can be counted by assuming that all the oxidants added to the reaction are consumed. ${ }^{12}$ In this respect, the quantification of photocarrier number in a solid state polymer might also be regarded as possible simply by comparing the spectral intensities of carriers between the solution and solid polymer samples. However, such a procedure cannot be applied to conjugated polymers because their electronic structure is usually different between the solid and liquid phases due to interchain interactions in the solid phase, making direct comparison of the spectral intensities of carriers difficult. Deriving a relation between the density and spectral intensity of carriers in solid polymers is, thus, essentially difficult.

Generally, in photoinduced absorption (PIA) measurements, the absorption signals induced by photoexcitation are used to discuss the photophysics of photo-generated species. However, in the measurements, bleaching signals, defined by loss of the absorption signals in the ground state, can also be detected and provide information about the ground state components consumed by photoexcitation. In particular, bleaching signals have, recently, been employed to identify the ground state components consumed by photoexcitation ${ }^{13,14}$ or those by charge-injection ${ }^{13-17}$ for conjugated polymers. In this article, we propose a method to determine the density of photocarriers in polymer films under continuous- photoirradiation directly from PIA measurements. The method utilizes the bleaching signals from PIA measurements and the steadystate absorption signals induced by chemical oxidation in solution. The method is applied to regioregular poly(3-hexylthiophene) (P3HT), a representative conjugated polymer used for BHJ solar cells. P3HT is known to form solid films that have self-organized microcrystalline domains composed of polymer chains with strong interchain couplings. ${ }^{18,19}$ The interchain couplings cause the electronic states to differ 
between solid versus solution samples, ${ }^{19}$ and the difference manifests when comparing the spectral structure and absorption cross section between the samples. Such differences are indeed shown in Fig. 1, which displays the steady-state absorption spectra for a film and solution of P3HT that contain the same number of polymer chains per unit area.

The method we propose is used to determine the photocarrier density of P3HT under continuous photoirradiation in a blended film of P3HT and methanofullerene, [6,6]-phenyl C61 butyric acid methyl ester (PCBM), which is one of the most studied and used films for BHJ solar cell applications. The photocarrier density in the film is first determined for conditions of continuous laser irradiation at $473 \mathrm{~nm}$. The method is further extended into the conditions of simulated 1 sun solar irradiation and identified to give an appropriate value. The method in this study is based on a simple theory focusing on the proportion of reduction in spectral intensity involved by carrier generation. The method can also be easily applied to films and devices of other materials. Determination of carrier density via the method must be effective to evaluate a device performance and discuss the photophysics of conjugated polymers.

\section{METHODOLOGY FOR DETERMINING PHOTOCARRIER DENSITY}

The methodology used to determine the density of the photocarriers in the solid film using PIA measurements is described in this section. Briefly, the photocarrier density is determined by converting the spectral intensity of the carriers in solution into those in the film through the bleaching signals. This procedure consists of three steps and is described here for a case of hole (cation) carriers: (i) Examine the relationship between the spectral intensity and the number of carriers generated by chemical oxidation in solution. (ii) Examine the relationship between the spectral intensity of the carriers in the solution and film samples. (iii) Convert the spectral intensity of the carriers in the solution into those in the film.

For the first step, the changes in the absorption spectrum induced by chemical oxidation must be quantified. Conjugated polymers can be oxidized in solution using

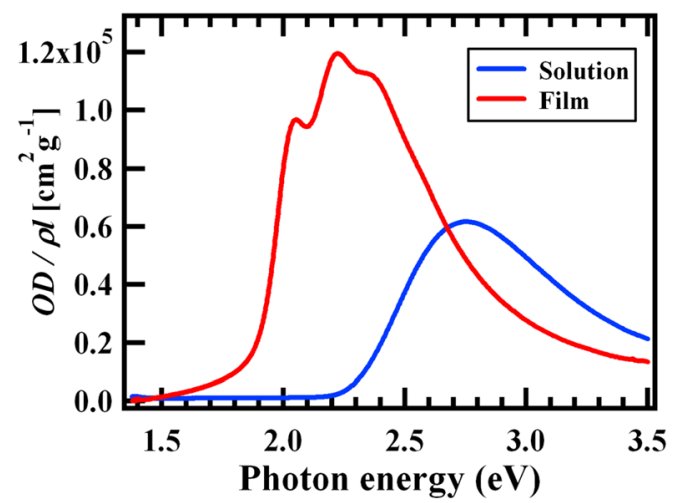

FIG. 1. Absorption spectra of a poly (3-hexylthiophene) (P3HT) solution and film divided by the product of the density or concentration $\rho$ of P3HT and path length $l$ for the absorption measurements: $\rho=1.15 \mathrm{~g} / \mathrm{cm}^{3}$ was used for the film. The result demonstrates that the absorption cross section is different between the solution and film samples. chemical reagents to generate cations. ${ }^{12,20,21}$ For the oxidation reaction, it can be assumed that all added oxidants are consumed unless the reagent is added in excess. The number of cation-carriers in solution is therefore quantified using the amount of added reagents. The changes in the carrier number are then monitored by observing the reduced intensity of the absorption signals in the ground state.

For the second step, a method to associate the carrier density of film with that of the solution is examined. The electronic states in conjugated polymers are usually different between the liquid and solid phases, and these differences appear in their absorption spectra. However, despite the differences in the electronic structure, the total number of electrons constituting the polymers is conserved between the liquid and solid phases as long as the number of polymer chains present in the solid and liquid phases is the same. We then consider the case in which the same number of carriers is generated in the liquid and solid phases with the same polymer number per area, as illustrated in Fig. 2. In this case, the proportion of polymers converted into carriers for all of the polymers is the same between the liquid and solid phases. Subsequently, we can obtain the next relationship

$$
\left(\frac{-\Delta O D_{g}}{O D_{g}}\right)_{s}=\left(\frac{-\Delta O D_{g}}{O D_{g}}\right)_{f}
$$

where $O D_{g}$ is the optical density of the ground state absorption and the indexes $s$ and $f$ are used for the solution and film, respectively. The quantity $-\triangle O D_{g}$ corresponds to the intensity of the bleaching signals and is proportional to the number of polymers converted into carriers. In this relationship, only the spectral intensity of the ground state absorption is used; the intensity of generated carriers is not used. The relation is based on the simple theory that the proportion of decrease in ground state absorption signals is the same throughout solution and film samples when the numbers of polymer chains and carriers are the same. The relation, thus, holds true regardless of degree of inter-chain interaction in the solid film. Moreover, it can be used for a case that temperature of samples varies by photoirradiation, as long as the ground state absorption is properly measured under the same photoirradiation intensity. However, it is noted that this

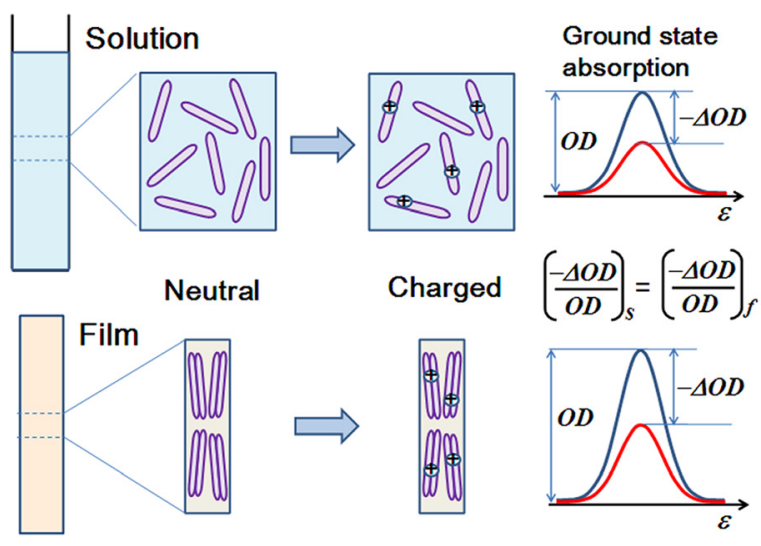

FIG. 2. Schematic drawings of the solutions and films of polymers with the same number of polymer chains and carriers per unit area. 
treatment is valid when the cause of the ground state- bleaching is attributable only to generation of carriers in the chemical oxidation and photoexcitation. Additionally, even if some morphological components coexist in the film, Eq. (1) is valid when carriers are equally generated from each morphological component; this factor can be judged by comparing a line shape between the bleaching and steady-state absorption spectra.

As the final step in determining the carrier density of film, the spectral intensity between the film and solution samples that have the same number of polymer chains per unit area is compared. The next relationship holds between the film and solution samples

$$
\rho_{f} l_{f}=C_{s} l_{s}
$$

where $\rho_{\mathrm{f}}$ and $C_{\mathrm{s}}$ are the polymer density for the film and the polymer concentration in the solution, respectively. Additionally, $l_{\mathrm{f}}$ is the film thickness and $l_{\mathrm{s}}$ is the optical path length during the absorption measurements in solution.

In the actual measurements, the quantity $\left(-\triangle O D_{g} / O D_{g}\right)_{f}$ is measured from the PIA measurements for a film, whose photocarrier density is unknown. As described above, solutions and films with the same number of carriers give the same values of $-\triangle O D_{g} / O D_{g}$ when they have the same number of polymer chains. We then define a virtual solution that is supposed to give the same value of $-\triangle O D_{g} / O D_{g}$ and have the same number of polymer chains per unit area as the actual film. Finally, by calculating the carrier number of the virtual solution from the relationship obtained in step (i) and converting it to the film, the carrier density of the film is determined. The actual determination of the density is shown in Sec. IV based on experimental results.

\section{EXPERIMENTAL}

P3HT was purchased from Merck Chemicals (Lisicon SP001: Regio-regularity 94\%) and was used as received. The chemical oxidation of P3HT was performed using anhydrous $\mathrm{FeCl}_{3}$ (Aldrich) as an oxidant. The solutions of P3HT and $\mathrm{FeCl}_{3}$ were prepared in anhydrous dichlorobenzene. The oxidation reaction was enacted by adding the $\mathrm{FeCl}_{3}$ solution to the P3HT solution, and the subsequent changes in the absorption spectrum were recorded immediately after adding the $\mathrm{FeCl}_{3}$ using a commercial ultraviolet-visible absorption spectrometer. The number of cations in the P3HT generated by the oxidation reaction was calculated from half of the amount of $\mathrm{FeCl}_{3}$ added because two equivalents of $\mathrm{FeCl}_{3}$ are necessary for a one-electron oxidation. ${ }^{22-25}$

The P3HT:PCBM film was fabricated by spin-casting a $17 \mathrm{mg} / \mathrm{ml}$ dichlorobenzene solution with a 1:1 mass ratio. The film was then annealed at $130^{\circ} \mathrm{C}$ for $25 \mathrm{~min}$ under vacuum. The PIA measurements were performed using a diodepumped solid-state (DPSS) laser with a cw- $473 \mathrm{~nm}$ output (CNI) for excitation (beam size: $2 \mathrm{~mm}$ ). The probe light was produced using a tungsten/halogen lamp and measured with a Si photodiode. The intensity of probe light hardly changed by irradiation of the excitation laser under intensities used in this study, suggesting that changes in the ground state absorption spectrum by the photoirradiation are negligible. The PIA setup detected small PL signals as a background. The contribution of PL was evaluated by measuring signals without the probe beam. The laser output was modulated with an acoustic-optic (AO) modulator at $700 \mathrm{~Hz}$, and the components of the probe light synchronized with the modulator were detected using a phase-sensitive lock-in technique. The intensity dependence of PIA and PL signals was measured by changing a bias for the AO modulator. All measurements were performed at room temperature under an inert atmosphere.

\section{RESULTS AND DISCUSSION}

First, the relationship between the concentration of cation carriers generated by chemical oxidation and the reduction in the spectral intensity of the ground-state absorption is examined for a P3HT solution. The absorption spectra of P3HT solution before and after adding $\mathrm{FeCl}_{3}$ used as an oxidizer are shown in Fig. 3. The absorption peak at $2.7 \mathrm{eV}$ of the P3HT solution decreases after adding $\mathrm{FeCl}_{3}$, and a new peak appears at approximately $1.5 \mathrm{eV}$. The new peak is similar to that of the P3HT cations in solution reported previously $^{7}$ and assigned to the cationic carriers. Fig. 3 also displays the absorption spectrum of the $\mathrm{FeCl}_{3}$ solution with the same concentration as that of the P3HT solution after adding $\mathrm{FeCl}_{3}$. The absorption peak at approximately $3.7 \mathrm{eV}$ observed from the $\mathrm{FeCl}_{3}$ solution is absent in the $\mathrm{P} 3 \mathrm{HT}$ spectrum after adding $\mathrm{FeCl}_{3}$. Therefore, the added $\mathrm{FeCl}_{3}$ can be regarded as being completely consumed by oxidation reactions. From the amount of consumed $\mathrm{FeCl}_{3}$, cation carriers generated were calculated to be approximately one per 10.5 thiophene rings. By analyzing the change in the spectral intensity after the oxidation, the next equation was obtained

$$
n_{s}=\frac{0.17 C_{s} N_{A}}{M_{w u}}\left(\frac{-\Delta O D_{g}}{O D_{g}}\right)_{s} .
$$

Here, $n_{\mathrm{s}}$ is the concentration of carriers in solution, $N_{\mathrm{A}}$ is Avogadro's number, and $M_{\mathrm{wu}}$ is the molecular weight per one molecular unit of P3HT chain (ca. 166). This equation represents the relationship between the carrier concentration and the spectral change for the P3HT solution.

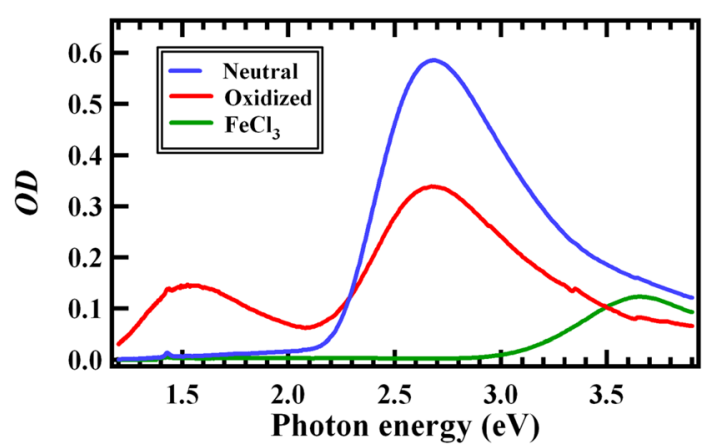

FIG. 3. Absorption spectra of the P3HT solution before (blue) and after (red) adding $\mathrm{FeCl}_{3}$ used as an oxidant. The green curve is the absorption spectrum of the $\mathrm{FeCl}_{3}$ solution with the same concentration as the P3HT solution after adding $\mathrm{FeCl}_{3}$. 
Subsequently, we define a virtual P3HT solution with the same number of polymer chains and carriers per unit area as the P3HT film used in the PIA measurements. The virtual solution is combined with the film by Eqs. (1) and (2) and satisfies Eq. (3). Therefore, using a value of $\left(-\triangle O D_{g} / O D_{g}\right)_{f}$ obtained from PIA measurements for the film, the carrier concentration of the virtual solution $n_{s, v}$ is given by the next equation

$$
n_{s, v}=\frac{0.17 \rho_{f} N_{A}}{M_{w u}} \frac{l_{f}}{l_{s}}\left(\frac{-\Delta O D_{g}}{O D_{g}}\right)_{f} .
$$

Finally, $n_{s, v}$ is converted to the density of carriers for the film $n_{\mathrm{f}}$. The number of carriers per unit area in the solution is given by $n_{s, v} l_{\mathrm{s}}$. When the solution and film have the same number of carriers per area, the relation $n_{s, v} l_{\mathrm{s}}=n_{\mathrm{f}} l_{\mathrm{f}}$ is valid. Therefore, $n_{\mathrm{f}}$ is obtained by the next equation

$$
n_{f}=\frac{0.17 \rho_{f} N_{A}}{M_{w u}}\left(\frac{-\Delta O D_{g}}{O D_{g}}\right)_{f} .
$$

The treatment described above is actually applied to the P3HT: PCBM blend film. For the blend film, we can assume that excitons generated in P3HT by photoexcitation are all converted into carriers due to highly efficient chargeseparation at the interface of P3HT and PCBM when the film is annealed. ${ }^{4,5,10}$ The PIA spectrum for the P3HT: PCBM blend film under photoexcitation of $580 \mathrm{~mW} / \mathrm{cm}^{2}$ is displayed in Fig. 4(a). The spectrum exhibits upward and downward $-\triangle O D$ peaks that are assigned to the photoinduced bleaching and absorption signals, respectively. The absorption signals arise from the photocarriers generated through
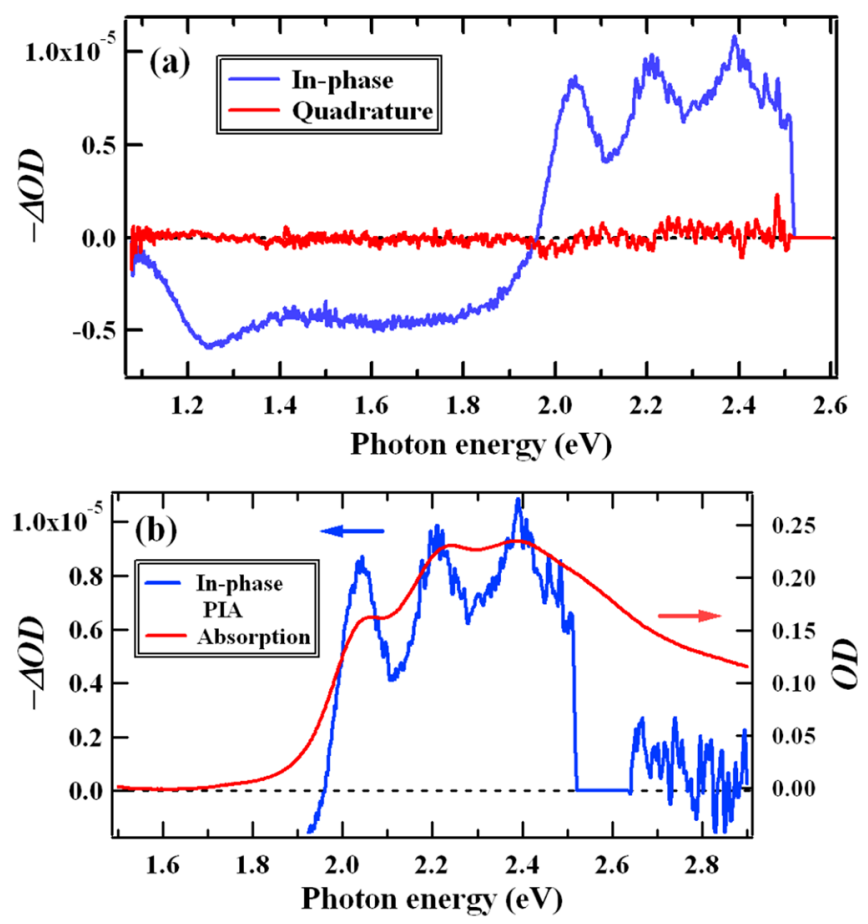

FIG. 4. (a) PIA spectrum for the P3HT:PCBM film measured under a 580 $\mathrm{mW} / \mathrm{cm}^{2}$ photoexcitation density. The excitation energy was $2.62 \mathrm{eV}$ $(473 \mathrm{~nm})$. (b) Comparison of the in-phase PIA spectrum and the ground state absorption spectrum for the P3HT:PCBM film. charge transfers from the P3HT excitons into the PCBM. The absorption peaks observed at approximately 1.3 and $1.8 \mathrm{eV}$ are assigned to the localized and delocalized positive polaron carriers, as previously reported. ${ }^{26-28}$ The bleaching signals occur due to loss of ground state absorption induced by photoexcitation. In this case, the bleaching signals are regarded as resulting from the generation of positive carriers in P3HT because all of the photoexcitations can be assumed to be converted into photocarriers in the film.

The ground state absorption spectrum for the P3HT:PCBM film is presented in Fig. 4(b). Comparing the absorption spectrum with the PIA-bleaching, one reveals that both spectra have three vibronic peaks at similar energy positions between 2.0 and $2.5 \mathrm{eV}$ and exhibit similar line shapes except that above $2.7 \mathrm{eV}$, where the ground state-absorption could be dominated by contributions of unaggregated molecules or short oligomers ${ }^{29}$ and bleaching signals are usually not observed. ${ }^{13,14}$ The similarity in the line shape below $2.7 \mathrm{eV}$ suggests that, even if there exist some morphological components in the film, carriers are equally generated from each morphological component. Therefore, the factor $\left(-\triangle O D_{g} / O D_{g}\right)_{f}$ determined from the results in Fig. 4 properly represents the proportion of carriers generated by photoexcitation and can be applied to Eq. (1).

By comparing the intensity of the ground state-absorption and bleaching spectra, $\left(-\triangle O D_{\mathrm{g}} / O D_{g}\right)_{f}$ was calculated to be $4.1 \times 10^{-5}$. We here note that, to obtain the carrier density under continuous photoirradiation, the PIA intensity after reaching the steady-state must be used. The observed PIA signals exhibited nearly a square-wave response in time-domain signals for the modulation at the measured frequency $(700 \mathrm{~Hz})$. The above value of $\left(-\Delta O D_{\mathrm{g}} / O D_{g}\right)_{f}$ was obtained using the magnitude of raw lock-in output for $-\triangle O D_{\mathrm{g}}$ and thus corrected by calibration data for square-wave voltages. After the correction, the $\left(-\triangle O D_{\mathrm{g}} / O D_{g}\right)_{f}$ was determined to be $1.0 \times 10^{-4}$. This value indicates that the proportion of P3HT converted into photocarriers during continuous photoirradiation is approximately $0.01 \%$ under the used photoexcitation intensity $\left(580 \mathrm{~mW} / \mathrm{cm}^{2}\right)$. Using the value of $\left(-\Delta O D_{\mathrm{g}} / O D_{g}\right)_{f}$ and $\rho_{\mathrm{f}} \sim 0.61 \mathrm{gcm}^{-3}$ estimated by assuming the density of P3HT and PCBM in films to be 1.15 and $1.3 \mathrm{~g} \mathrm{~cm}^{-3}$, respectively, ${ }^{30}$ the photocarrier density $n_{\mathrm{f}}$ under the photoexcitation intensity is finally determined to be $3.5 \times 10^{16} \mathrm{~cm}^{-3}$ using Eq. (5).

We have now determined the photocarrier density under monochromatic photoexcitation at $473 \mathrm{~nm}$ based on the method developed here. Hereafter, we shall propose a procedure to estimate the photocarrier density under simulated solar irradiation from the determined density and compare the estimated density with that reported previously from another method.

Generally, the generation process of photocarrier is described by the following bimolecular rate equation with $n$ being the carrier density:

$$
\frac{d n}{d t}=G-\beta n^{2},
$$

where $G$ and $\beta$ are the generation rate and bimolecular recombination rate constants of the photocarrier, respectively. 
In the steady state, the carrier density $n$ is given by $(G / \beta)^{1 / 2}$ and corresponds to $n_{f}$. In the polymer: fullerene blend system, photocarriers are generated via singlet excitons in polymers generated by photoexcitation. $G$ is then expected to increase linearly for the increase of photoexcitation intensity $I$, since the density of singlet excitons is proportional to $I$. Therefore, $n_{f}$ is expected to scale with $I^{1 / 2}$ unless $\beta$ depends on the photoexcitation intensity.

The $I$-dependence of PIA and PL intensity was examined and is shown in Figs. 5(a) and 5(b), including the results of fit using a function of $I^{a}$. The best-fit result for the PL intensity was $a=1.2$, indicating an almost linear increase of singlet excitons for increasing $I$. In contrast, the PIA intensity measured at $1.30 \mathrm{eV}$ was proportional to $I^{0.57}$. This demonstrates that P3HT carriers generated by photoexcitation increase approximately in proportion to $I^{1 / 2}$. This relation also indicates that $\beta$ is almost independent of the excitation intensity under continuous photoirradiation. Fig. 5(b) shows that bleaching signals measured at $2.48 \mathrm{eV}$ increase in proportion to $I^{0.56}$, quite similar to the $I$-dependence of the carrier signals. The similarity in the $I$-dependence indicates a complete correspondence between the carrier and bleaching signals, suggesting that the final nature of photo-generated species in P3HT can be entirely regarded as carriers, due to very high conversion efficiencies in the annealed P3HT:PCBM film.

The observed similarity in $I$-dependence between carrier and bleaching signals allows us to calculate the $I$-dependence of photocarrier density in $\mathrm{P} 3 \mathrm{HT}$ under continuous photoirradiation. The result of calculation is shown in the right axis of Fig. 5(b). The calculated I-dependence of photocarrier
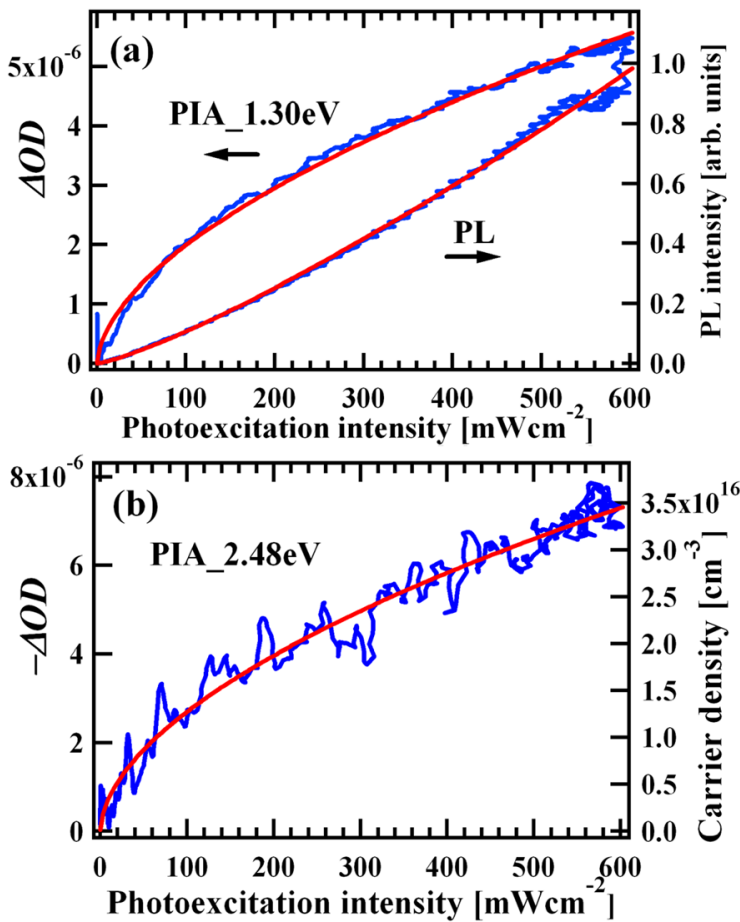

FIG. 5. (a) Photoexcitation intensity (I)-dependence of the PIA signals measured at $1.30 \mathrm{eV}$ (carrier absorption) and PL signals. Fitted curves are results of fit using a function of $I^{a}$. The best fit results were $a=0.57$ and 1.2 for the PIA and PL signals, respectively. (b) I-dependence of the PIA signals at $2.48 \mathrm{eV}$ (bleaching). The best fit result was $a=0.56$. The right axis indicates the photocarrier density calculated from the PIA signals. density was obtained from the P3HT:PCBM film with a thickness of $32 \mathrm{~nm}$. We here attempt to obtain a formula to calculate the photocarrier density for arbitrary thickness of film using the result of the $32 \mathrm{~nm}$-film. In the observed trend of the photocarrier density being proportional to $I^{1 / 2}$, the density of absorbed photon in the film is expected to simply increase linearly depending on $I$. It can thus be assumed that the photocarrier density is proportional to the square root of the density of absorbed photon in the film. Using the relation for the absorbed photon, the photocarrier density $n_{\mathrm{c}}$ of annealed P3HT:PCBM films for arbitrary photoexcitation intensity $I\left(\mathrm{Wcm}^{-2}\right)$ and thickness $l_{f}(\mathrm{~cm})$ is given as follows:

$$
n_{c}=1.5 \times 10^{14} \sqrt{\frac{I\left\{1-\exp \left(-1.1 \times 10^{5} l_{f}\right)\right\}}{l_{f}}}\left[\mathrm{~cm}^{-3}\right] \text {. }
$$

This formula was obtained for monochromatic excitation at $473 \mathrm{~nm}$. The same procedure can be generalized for simulated solar light under Air Mass (AM) 1.5 (1 sun) conditions $\left(100 \mathrm{~mW} / \mathrm{cm}^{2}\right)$, by using the relation of photocarrier density being proportional to the square root of the density of absorbed photons for the sun light. The spectrum of incident photon number per area calculated from the solar power spectrum under the 1 sun condition is shown in Fig. 6(a). Use of the photon number spectrum and the ground state absorption spectrum enables to calculate the spectrum of density of absorbed photons per second. The result of calculation for the $32 \mathrm{~nm}-$ P3HT: PCBM film is shown in Fig. 6(b). Moreover, the integral of the spectrum gives the total photon density per second, which was determined to be
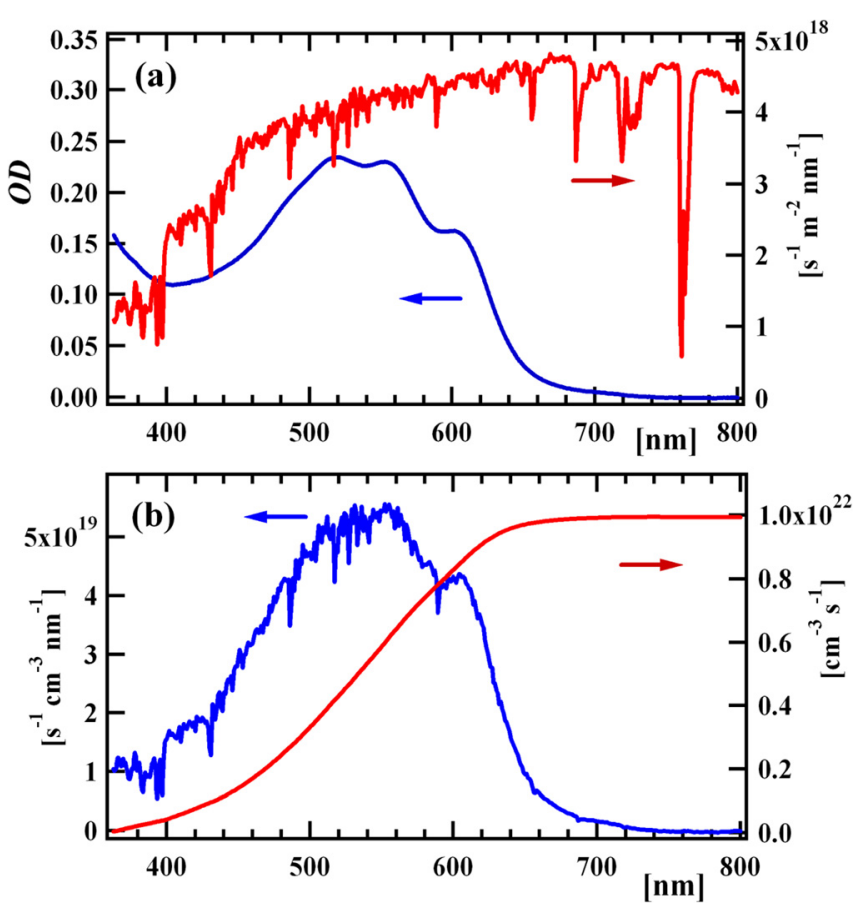

FIG. 6. (a) Comparison of the steady-state absorption spectrum of the P3HT: PCBM film (left axis) and spectrum of incident photon number per area calculated from the solar power spectrum under the 1 sun condition (right axis). (b) The density spectrum of absorbed photons per second for the P3HT: PCBM film (left axis) and integral of the density spectrum (right axis). 
$9.9 \times 10^{21} \mathrm{~cm}^{-3} \mathrm{~s}^{-1}$. The density of absorbed photons for the $580 \mathrm{~mW} / \mathrm{cm}^{2}$ - excitation at $473 \mathrm{~nm}$ is calculated to be $4.3 \times 10^{23} \mathrm{~cm}^{-3} \mathrm{~s}^{-1}$ for the film. From these results, the photocarrier density under the 1 sun condition is determined to be $2.0 \times 10^{15} \mathrm{~cm}^{-3}$. This result can be extended into cases of films of arbitrary thickness as follows:

$$
n_{c}=6.6 \times 10^{12} \sqrt{\frac{1-\exp \left(-1.1 \times 10^{5} l_{f}\right)}{l_{f}}}\left[\mathrm{~cm}^{-3}\right]
$$

This equation enables a simple estimate of mean photocarrier density of P3HT generated in annealed P3HT: PCBM film. It corresponds to half of the maximum photocarrier density that can be used in solar cell operation when assuming that P3HT and PCBM provide the same density of photocarriers. The carrier density of annealed P3HT: PCBM solar cells was recently measured by charge extraction measurements. ${ }^{31,32}$ From the reported results, the photocarrier density under the 1 sun condition for the cells with about $200 \mathrm{~nm}$ thickness of films is estimated to be approximately $3-4 \times 10^{15} \mathrm{~cm}^{-3}$. Half of the value, corresponding to the density of P3HT, is very close to the photocarrier density calculated from Eq. (8) for $l_{f}=200 \mathrm{~nm}: 1.4 \times 10^{15} \mathrm{~cm}^{-3}$. This indicates that the method used in this study gives an appropriate value of photocarrier density consistent with previous reports. In the present method, all photocarriers including non-mobile carriers can be counted in the estimate because the method is based on the PIA signals, whereas the charge extraction measurements count the carrier density of mobile carriers. Therefore, the close value of photocarrier density suggests that the photocarriers generated in annealed P3HT: PCBM films are mostly used as mobile carriers.

We finally emphasize that the equations and procedures obtained in this study can also be applied to estimating the carrier density in transient absorption (TA) measurements and spectroscopic measurements of charge injection when the bleaching signals are properly measured. The intensity of TA signals at $970 \mathrm{~nm}(1.28 \mathrm{eV})$ for the P3HT: PCBM film was previously converted to the photocarrier density based on the magnitude of extinction coefficient estimated considering the magnitude of transient photocurrent data. ${ }^{33}$ However, we note that there are two types of carriers in P3HT films, DPs and LPs, as shown in the PIA spectrum in Fig. 4(a), and they are regarded as independent of each other. Both carriers can contribute to a photocurrent, and hence the proportion of carriers responsible for the TA signals must be properly estimated when comparing the carrier signals with the photocurrent data: particularly, the relative ratio of carrier signals in the two types of carriers cannot be simply estimated. Therefore, determination of carrier density directly from carrier signals in spectroscopic measurements is essentially difficult to P3HT. In the present research, we used bleaching signals to estimate the photocarrier density. The bleaching signals include both contributions of DPs and LPs and are not affected by the composition ratio of them. Therefore, the method in this study can give a reliable estimate from spectroscopic signals and can also be applied to other materials with two or more types of carriers as in
P3HT. Moreover, the advantageous point of the present method is that it enables an estimate of carrier density independent of current measurements. Therefore, combined applications of the present method and methods via current measurements can be applied to studying roles of nonmobile carries such as trapped carriers and charge-transfer carrier pairs.

\section{CONCLUSIONS}

We proposed a method to determine the density of photocarriers generated under continuous photoirradiation from spectroscopic measurements using PIA measurements. The bleaching signals from the PIA measurements and the absorption signals of the cations generated by chemical oxidation in solution were employed to determine the photocarrier density. This method was applied to P3HT in a P3HT:PCBM film. The photocarrier density was determined to be $3.5 \times 10^{16} \mathrm{~cm}^{-3}$ in the steady state under the applied photoexcitation intensity $\left(580 \mathrm{~mW} / \mathrm{cm}^{2}\right)$. The carrier density was identified to increase nearly in proportion to the square root of photo-excitation intensity. Using the relation, we determined a formula to estimate the photocarrier density under simulated 1 sun solar irradiation for the P3HT: PCBM film of an arbitrary thickness. The equations determined here can also be applied for transient absorption measurements and spectroscopic measurements of charge injection when the bleaching signals are properly measured. Combined applications of the present method and methods via current measurements can lead to revealing roles of non-mobile carries such as trapped carriers and charge-transfer carrier pairs.

\section{ACKNOWLEDGMENTS}

This work was supported in part by a Grant-in-aid (No. 24656065) from the Japanese Ministry of Education, Culture, Sports, Science, and Technology.

${ }^{1}$ I. D. W. Samuel, G. Rumbles, and R. H. Friend, in Primary Excitations in Conjugated Polymers: Molecular Exciton Versus Semiconductor Band Model, edited by N. S. Sariciftci (World Scientific, Singapore, 1997), Chap. 7.

${ }^{2}$ E. M. Conwell, in Organic Electronic Materials, edited by R. Farchioni and G. Grosso (Springer-Verlag, Berlin, 2001), Chap. 4.

${ }^{3}$ D. Moses, in Ultrafast Dynamics and Laser Action of Organic Semiconductors, edited by Z. V. Vardeny (Taylor \& Francis, 2009), Chap. 3.

${ }^{4}$ B. C. Thompson and J. M. J. Fréchet, Angew. Chem. Int. Ed. 47, 58 (2008).

${ }^{5}$ S. Günes and N. S. Sariciftci, Inorg. Chim. Acta 361, 581 (2008).

${ }^{6}$ Z. L. Guan, J. B. Kim, H. Wang, C. Jaye, D. A. Fischer, Y. L. Loo, and A. Kahn, Org. Electron. 11, 1779 (2010).

${ }^{7}$ T. Liu and A. Troisi, J. Phys. Chem. C 115, 2406 (2011).

${ }^{8}$ L. H. Li, O. Y. Kontsevoi, and A. J. Freeman, J. Phys. Chem. C 118, 10263 (2014).

${ }^{9}$ C. F. N. Marchiori and M. Koehler, J. Phys. D 47, 215104 (2014).

${ }^{10}$ R. Long and O. V. Prezhdo, Nano Lett. 14, 3335 (2014).

${ }^{11}$ T. M. Clarke and J. R. Durrant, Chem. Rev. 110, 6736 (2010) and references therein.

${ }^{12}$ M. J. Nowak, S. D. D. V. Rughooputh, S. Hotta, and A. J. Heeger, Macromolecules 20, 965 (1987).

${ }^{13}$ K. Kanemoto, M. Yasui, D. Kosumi, A. Ogata, M. Sugisaki, T. Karasawa, I. Akai, and H. Hashimoto, Phys. Rev. Lett. 103, 187402 (2009).

${ }^{14}$ K. Kanemoto, M. Yasui, T. Higuchi, D. Kosumi, I. Akai, T. Karasawa, and H. Hashimoto, Phys. Rev. B 83, 205203 (2011). 
${ }^{15}$ H. Sirringhaus, P. J. Brown, R. H. Friend, M. M. Nielsen, K. Bechgaard, B. M. W. Langeveld-Voss, A. J. H. Spiering, R. A. J. Janssen, E. W. Meijer, P. Herwig, and D. M. de Leeuw, Nature 401, 685 (1999).

${ }^{16}$ P. J. Brown, H. Sirringhaus, M. Harrison, M. Shkunov, and R. H. Friend, Phys. Rev. B 63, 125204 (2001).

${ }^{17}$ P. J. Brown, D. S. Thomas, A. Köhler, J. S. Wilson, J. S. Kim, C. M. Ramsdale, H. Sirringhaus, and R. H. Friend, Phys. Rev. B 67, 064203 (2003).

${ }^{18}$ R. D. McCullough, S. Tristram-Nagle, S. P. Williams, R. D. Lowe, and M. Jayaraman, J. Am. Chem. Soc. 115, 4910 (1993).

${ }^{19}$ T. A. Chen, X. M. Wu, and R. D. Rieke, J. Am. Chem. Soc. 117, 233 (1995).

${ }^{20}$ J. A. E. H. van Haare, E. E. Havinga, J. L. J. van Dongen, R. A. J. Janssen, J. Cornil, and J.-L. Brédas, Chem. Eur. J. 4, 1509 (1998).

${ }^{21}$ N. Sumi, H. Nakanishi, S. Ueno, K. Takimiya, Y. Aso, and T. Otsubo, Bull. Chem. Soc. Jpn. 74, 979 (2001).

${ }^{22}$ D. Fichou, G. Horowitz, B. Xu, and F. Garnier, Synth. Met. 39, 243 (1990).

${ }^{23}$ D. Fichou, B. Xu, G. Horowitz, and F. Garnier, Synth. Met. 41, 463 (1991).
${ }^{24}$ J. Guay, P. Kasai, A. Dim, R. Wu, J. M. Tour, and L. H. Dao, Chem. Mater. 4, 1097 (1992).

${ }^{25}$ H. Nakanishi, N. Sumi, S. Ueno, K. Takimiya, Y. Aso, T. Otsubo, K. Komaguchi, M. Shiotani, and N. Ohta, Synth. Met. 119, 413 (2001).

${ }^{26}$ R. Österbacka, C. P. Au, X. M. Jiang, and Z. V. Vardeny, Science 287, 839 (2000).

${ }^{27}$ X. M. Jiang, R. Österbacka, O. Korovyanko, C. P. An, B. Horovitz, R. A. J. Janssen, and Z. V. Vardeny, Adv. Funct. Mater. 12, 587 (2002).

${ }^{28}$ O. J. Korovyanko, R. Österbacka, X. M. Jiang, and Z. V. Vardeny, Phys. Rev. B 64, 235122 (2001).

${ }^{29}$ J. Clark, C. Silva, R. H. Friend, and F. C. Spano, Phys. Rev. Lett. 98, 206406 (2007).

${ }^{30}$ J. W. Kiel, B. J. Kirby, C. F. Majkrzak, B. B. Maranville, and M. E. Mackay, Soft Matter 6, 641 (2010).

${ }^{31}$ C. G. Shuttle, R. Hamilton, B. C. O'Regan, J. Nelson, and J. R. Durrant, Proc. Natl. Acad. Sci. U.S.A. 107, 16448 (2010).

${ }^{32}$ M. Gluecker, A. Foertig, V. Dyakonov, and C. Deibel, Phys. Status Solidi RRL 6, 337 (2012).

${ }^{33}$ C. G. Shuttle, B. O'Regan, A. M. Ballantyne, B. Nelson, D. D. C. Bradley, and J. R. Durrant, Phys. Rev. 78, 113201 (2008). 